\title{
Kilimanjaro porter spines
}

\author{
Marieke Cornelia Johanna Dekker ${ }^{1,2}$ Emma Sophie du Marchie Sarvaas ${ }^{3,4}$. Sakina Mehboob Rashid $\mathbb{C}^{4}$. \\ Mubashir Alavi Jusabani ${ }^{4}$. William Patrick Howlett ${ }^{5}$
}

Received: 22 November 2017 / Revised: 15 December 2017 / Accepted: 19 December 2017

(C) International Spinal Cord Society 2018

Following a recent study at our centre that examined traumatic spinal cord injuries (SCIs) admitted to our Orthopaedics Department, we were inspired to a further analysis of the local porter population working on Mount Kilimanjaro, the highest free-standing mountain in the world (5895 metres). On the African continent, manhandling heavy loads is customary from an early age onwards but in Kilimanjaro porters, one could query increased rates of spinal injury due to their demanding profession.

The maximum load allowed for by the Tanzanian mountain authorities is $20 \mathrm{~kg}$, although individual weights up to $32 \mathrm{~kg}$ have been reported. These weights are carried on the shoulders or head-often interchangeably-over several days (an average Kilimanjaro ascent is 6-8 days) and during long hours (Fig. 1). Porters accompany climbers up to the last refuge hut (Kibo Hut at $4705 \mathrm{~m}$ ) and their itinerary leads through rainforest onto highland and Alpine desert climate, with typical temperatures ranging from -6 to +25 degrees centigrade. Few data are available on the relation between spine or SCI and the porter profession, let alone dedicated patient series on spinal conditions in Kilimanjaro porters. The low reported incidence of traumatic spinal cord injury in porters elsewhere [1, 2] may illustrate that such sampling would be a very long-term project.

Marieke Cornelia Johanna Dekker

marieke@zwets.com

1 Department of Internal Medicine, Department of Pediatrics and Child Health, Kilimanjaro Christian Medical Centre, Moshi, Tanzania

2 Department of Neurology, Radboudumc Nijmegen, Nijmegen, Netherlands

3 University Medical Centre Groningen, Groningen, Netherlands

4 Kilimanjaro Christian Medical Centre, Moshi, Tanzania

5 Department of Internal Medicine, Kilimanjaro Christian Medical Centre, Moshi, Tanzania
The best-known areas of the world where heavy goods are carried up over rough terrain to extremely high altitudes by manpower are the Himalayas [3] and South-America [4]. A spinal surgeon who studied a series of injuries in Zimbabwe coined the condition 'Porter's Neck' that encompasses anything from pain and bony injury to quadriplegia and death owing to presumed acute intervertebral disk herniation and cord compression [1]. This Zimbabwean study comprised a live experiment where volunteers underwent serial spinal X-rays while receiving a 90.7 kilogram $(\mathrm{kg})$ bag of maize on their head, showing real time and significant compression of the vertebral column [1]. The reported spontaneous incidence of this kind of injury, however, was very low, with only 14 cases over 10 years [1] and another South-African study mentioning 12 cases over 13 years time [2].

There is an unpublished study on musculoskeletal complaints in the Kilimanjaro porter population, where back and joint pain scores are comparable with the control population of shopkeepers in Moshi town (with permission: Cyr et al., unpublished work) [5], a finding that could be attributed to some methodological issues as well as to a higher pain perception threshold in the physically demanding porter profession.

Studies on porter-related medical problems elsewhere, such as in Nepal [3] cover a wide range of common health problems, of which interestingly both high altitude illnesses and spinal problems only constituted a minor section.

We are well aware of limitations in our study design and methods, restricted by small numbers, lack of dedicated registries, and restricted resources; however, we felt it would be interesting to have inventory of porter-specific spinal problems despite the fact that our setting and facilities did not allow for more robust methodology.

At our centre, Kilimanjaro Christian Medical Centre (KCMC), 20 kilometres from the summit as the crow flies and harbouring the nearest-by Intensive Care and Neurology and Orthopaedic specialist clinics, one of the authors (WP Howlett) collected data on neurological disease 


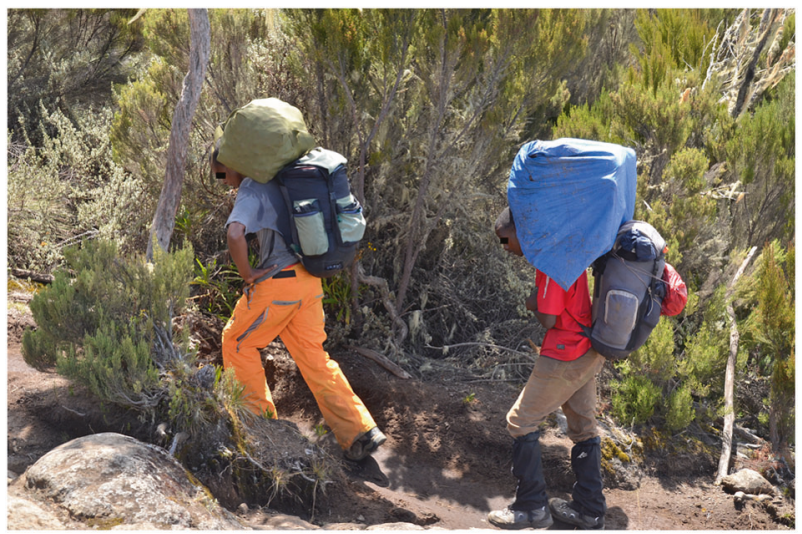

Fig. 1 Porters carrying heavy loads to facilitate climbers' ascent of Mount Kilimanjaro

patterns with a special interest into morbidity and mortality in Kilimanjaro climbers and porters $(n=2040)$. Likewise, a register of spinal cord injuries for the Orthopaedic Rehabilitation Unit was available $(n=125)$. A cumulative total of 2165 charts were reviewed at our centre. We expected that porters suffering from acute conditions would be admitted to the Orthopaedics ward and those with more chronic conditions would pass through the Medical clinics and wards. In addition, we consulted medical professionals working or having worked in health centres nearby the Kilimanjaro National Park gates along the mountain's circumference in the past three decades. A 7-year observation period from a hospital near the busiest trail (G. Hjorth, personal communication) was available, albeit not a systematic registry.

A breakdown into any spinal cord pathology in the large hospital-based general neurological patient series in 2040 individuals shows 297 cases of paraplegia. In this medical, non-traumatic paraplegia category, very few cases of syringomyelia were observed, most being females and no porters among them. A series of 125 traumatic SCI patients admitted to the Orthopaedics Department at KCMC comprised no porters. Orthopaedic surgeons, physicians, and mountain expedition doctors working in the Kilimanjaro foothills, representing cumulative decades of experience, reported to have never observed spinal injury, and very rarely other head and neck pains in any porter.

There are a number of possible causes of misclassification. Under-reporting owing to logistic reasons is potentially important. Mountain rescue infrastructure is basic, and many fatalities are not transported to our hospital owing to distance and cost. Porters tend to go to cheaper healthcare facilities although many employers will enable assessment in a referral hospital if need be. Health complaints may also go unreported for fear of losing one's job. Purely surgical or orthopaedic injury (unless they involve spinal cord) will also bypass medical assessment. However, they were not found in the spinal cord registers nor could consulted orthopaedic surgeons recollect any pathology in porters except for ankle and leg fractures.

We would expect that porters with SCI like para- or tetraplegia, chronic spinal conditions such as intervertebral disc herniation, stenosis and syringomyelia as well as significant back- and neck ache with or without irradiating pain, would have been referred to our hospital. Amongst our SCI series and from long-term (2007-2013) paraplegia registers in the medical ward of our hospital, there was not one Kilimanjaro porter. The intriguing absence of significant spinal conditions is also observed in Himalayan porters [3].

In our Tanzanian general neurology and orthopaedics clinics, chronic back and neck pain, and lumbosacral or cervical radicular/pseudo-radicular syndromes are uncommon. They are also absent from Africa's list of leading chronic health conditions in a recent global burden of disease survey [6]. In higher income countries, however, they form a major, if not leading source of neurological consultations claiming a significant share of a neurologist's outpatient care [6]. In this literally high-burden profession, a stronger spinal constitution per se can be another possible explanation, as it could be elsewhere in Sub-Sahara Africa.

Whether the genetic make up of Kilimanjaro porters also has a role in the observed lack of work-related complaints is a potential area of further research. The extent to which such findings may explain the lack of spine related complaints in the porter population may be quite variable.

In addition, frequent load-carrying may have strengthened the para-spinal muscles of the porters, thus providing a protective effect against prolonged disc compression by supporting the vertebral column. A comparison between the porters and a control population with focus on differences in muscle density and posture maintained, whereas weight bearing would also be an interesting study.

The environment of Mount Kilimanjaro and the working conditions of the porters represent a unique opportunity to study the epidemiological relationship between duration of work, amount of weights carried and the nature of resultant symptoms. Furthermore, the distinctive working conditions of Kilimanjaro porters are matched by only a few regions in the world, thus setting the groundwork for a collaboration between researchers in geographically similar locations around the world.

In spite of a relatively spared spine, porters on Africa's highest mountain have one of the world's extreme professions and thereby other work-related health risks. Absence of hard figures does not allow for quantitative conclusions, illustrating the importance of systematic quantitative data collection. 


\section{Compliance with ethical standards}

Conflict of interest The authors declare that they have no conflict of interest.

\section{References}

1. Levy LF. Porter's neck. BMJ. 1968;2:16-19.

2. Scher AT. Injuries to the cervical spine sustained while carrying loads on the head. Paraplegia. 1978;79:94-101.

3. Basnyat B, Litch JA. Medical problems of porters and trekkers in the Nepal Himalaya. Wilderness Environ Med. 1997;8:78-81.
4. Bauer IL. Inca trail porters: the health of local tourism employees as a challenge for travel medicine. J Travel Med. 2003;10:94-99.

5. Cyr J, Berta J, Bourgois M, Erdan T, Gourlay E, Mtuy T, et al. An investigation of musculoskeletal symptoms in porters on Mount Kilimanjaro. (Unpublished, 2016).

6. Hay SI, Jayaraman SP, Truelsen T, Sorensen RJ, Millear A, Giussani G, et al. GBD 2015 Disease and Injury Incidence and Prevalence Collaborators. Global, regional, and national incidence, prevalence, and years lived with disability for 310 diseases and injuries, 1990-2015: a systematic analysis for the Global Burden of Disease Study 2015. Lancet. 2016;388:1545-602. 